The goal of the Federal Motor Carrier Safety Administration (FMCSA) is to reduce the number and severity of large truck-involved crashes through more commercial motor vehicle and operator inspections and compliance reviews, stronger enforcement measures against violators, expedited completion of rulemaking proceedings, scientifically sound research, and effective commercial driver's license testing, recordkeeping, and sanctions. The Office of Research and Technology manages research and technology development and deployment programs for the FMCSA.

There are nine major research and technology focus areas: crash causation and risk factors; regulatory evaluation and reform; compliance and enforcement; HAZMAT safety and cargo tank integrity; carrier safety management; driver training and performance enhancement; driver alertness and fatigue; driver physical qualifications; and car-truck proximity.

Driver alertness and fatigue primarily supports current and future hours-of-service rulemaking activities, along with fatigue outreach and fatigue management technology development.

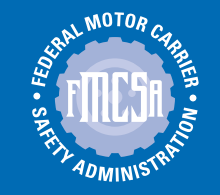

Office of Research and Technology 400 Seventh Street, SW MC-RT; Room 3107 Washington, DC 20590

\section{Stress and Fatigue Effects of Driving Longer-Combination Vehicles}

\section{Introduction}

Driver fatigue is a safety issue of special concern to commercial motor vehicle (CMV) transportation. Section 4007(d) of the Intermodal Surface Transportation Efficiency Act of 1991 directed the Department of Transportation to study, among other issues, the effects of longer-combination vehicle (LCV) operation on driver stress and fatigue. To accomplish this, the Federal Motor Carrier Safety Administration [formerly the Office of Motor Carriers, Federal Highway Administration (FHWA)] sponsored a study to find out if there were significant differences between the stress and fatigue effects of operating single tractor-trailer vehicles and tractors pulling triple-trailer combinations. The work was carried out in cooperation with the National Highway Traffic Safety Administration, by the Battelle Human Factors Transportation Center, in Seattle, Washington.

This study was conducted as a part of a major program by FHWA to investigate commercial driver fatigue in CMV operations. This tech brief summarizes the study final report, Stress and Fatigue Effects of Driving Longer-Combination Vehicles, which will be available from the National Technical Information Service.

\section{Purpose}

This study investigated the possibility that LCVs, with their increased length, greater weight, and greater number of trailers, could significantly increase the amount of stress and fatigue experienced by the driver. The study was limited to the issue of fatigue under proper and normal operating conditions; it was not intended as an investigation of the safety of these vehicles.

\section{Methodology}

Between October 31, 1994 and January 21, 1995, 24 experienced LCV drivers drove approximately $2700 \mathrm{mi}$ (4347 km) each in specially equipped and loaded single- and triple-trailer commercial vehicles under controlled experimental conditions. Tractors were equipped with video and digital equipment to gather data on the drivers' performance during the study. Drivers also answered standardized questionnaires concerning their perception of stress and fatigue at five scheduled times during the driving day.

Drivers were recruited from nearly a dozen companies to participate in the study, and they represented both large national fleets and smaller regional operations. The ages of participants ranged from 40 to 62 years; each reported at least 9 years of commercial motor vehicle driving experience. All had recent driving experience with tripletrailer combinations.

Three tractor-trailer combination vehicles were used in the study. Each driver who participated in the study was assigned a specific tractor and drove it for the entire study week, using it to alternately pull each of the three different trailer configurations: 
- single 48-foot trailer;

- A triple-trailer combination with three 28-foot trailers and standard converter dollies (A- dollies); and

- A triple-trailer combination with three 28-foot trailers and double-drawbar, self-steering converter dollies (C-dollies).

Standard A converter dollies differ from self-steering $C$ converter dollies in their design and method of attachment to the towing trailer (see Figure 1). The A-dolly consists of a fixed axle arrangement attached to the towing trailer at a single point, while the C-dolly consists of a self-steering axle arrangement attached to the trailer ahead by two connections spaced about 4 feet apart.

Each driver participated in the study for 7 consecutive days, beginning with a familiarization/training day on Sunday. All driving was done during daylight hours. Drivers encountered typical operating conditions and followed standard operating procedures. Each driving

Figure 1.

Plan view of converter dolly characteristics.

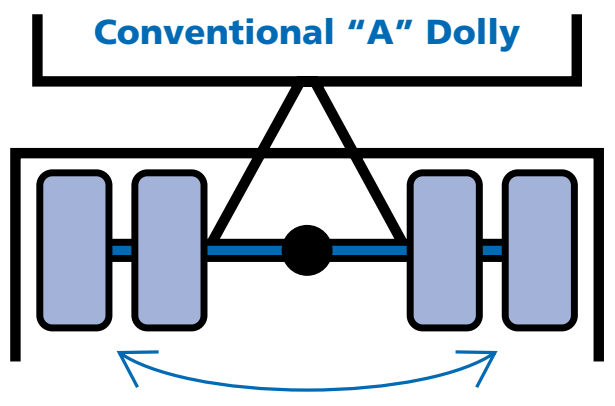

- Steering by single pivot point

- Single connection to lead trailer

- Solid Axle

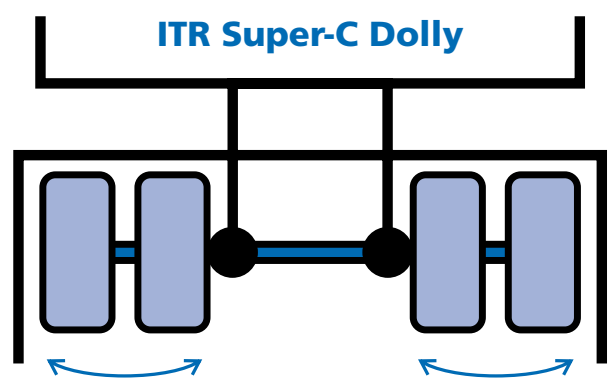

- Wheels steer individually

- Dual connection to lead trailer

- Self-steering Axle

Table 1.

Fatigue-related measures.

\begin{tabular}{ll|l}
\hline Type & Measure & Purpose \\
\hline \multirow{2}{*}{ Self Report } & Stanford Sleepiness Scale & Perceived fatigue \\
\cline { 2 - 3 } & NASA Raw Task Load Index & Perceived workload \\
\cline { 2 - 3 } Computer & Worksafe Australia Questionnaire & Perceived change in fatigue level \\
\hline \multirow{2}{*}{ Probe Tests } & Critical Tracking Task & Hand-eye coordination \\
\cline { 2 - 3 } & Unprepared Simple Reaction Time & Reaction time \\
\hline & Two Finger Tapping Test & Motor coordination \\
\hline & Code Substitution Task & Perception and short-term memory \\
\hline \multirow{2}{*}{ Driving } & Lane Deviation Squared & $\begin{array}{l}\text { Changes in lane position weighted to highlight } \\
\text { large changes }\end{array}$ \\
\hline & Maximum Lane Deviation & Extent of change of lane position \\
\hline & Lane Standard Deviation & Variability of lane position \\
\hline & Large Steering Wheel Reversals & Number of large corrections to steering \\
\hline \multirow{2}{*}{ Physiological } & Heart Period & Physical and mental workload \\
\hline Heart Period Variability & Changes in physical and mental workload \\
\hline
\end{tabular}


day consisted of approximately 10 duty-hours, of which approximately 8 hours were spent operating the vehicle. The remaining duty-hours involved taking performance tests, responding to interviews, and other study-related activities. Drivers were given a 1-hour lunch break and two 15-minute rest breaks during the day.

Several different data collection methods were utilized during this study (see Table 1). The researchers collected background information from driver questionnaires and interviews, field observer notes, and notes made during informal sessions with participants. This information was used to help clarify the more formal data gathered during the study.

The data were used to answer two questions:

- Does LCV operation result in a statistically significant increase in driver stress and fatigue when compared with single tractor-trailer combinations?

- Are the trailer configuration result contributions to fatigue and performance excessive in terms of criteria for safe operations?

\section{Results}

The results of the study provide unambiguous answers to both of the preceding questions. First, with regard to triple-trailer versus single-trailer configuration differences in fatigue, analyses revealed a consistent pattern of statistically significant results across all variables. For example, trailer configurations and other task demand characteristics consistently affected performance (lane-keeping measures), subjective workload, and fatigue/physiological state. Configuration results across all variables generally followed a single, C-dolly, A-dolly (SCA) pattern, with C-dolly results intermediate to those for single and A-dolly configurations.

Fatigue/physiological recovery and subjective workload results also indicated configuration-related carryovers (next-day effects) that followed the SCA pattern (see Figure 2). Specifically, the day following the A-dolly showed a relatively high level of carryover fatigue and resulting workload increase, while the day following the C-dolly showed relatively neutral carryover effects. In contrast, positive recovery was seen the day following the single-trailer configuration. Lane-keeping carryover effects paralleled the SCA fatigue/physiological state and workload patterns. Lane variability significantly increased on the day following the driving of the A-dolly, increased moderately the day following the C-dolly, and decreased on the day following the single.

The study also provided insight regarding configuration contributions to fatigue and performance degradations in terms of two criteria for safe operation: 1) probability of lane exceedance; and 2) operator workload criteria. In terms of the first of these, the results indicated that C-dolly triple-trailer drivers would experience substantially less fatigue and a lower probability of lane exceedance than drivers of A-dolly triple-trailer configurations. Secondly, with regard to operator workload, the results indicated that average triple-trailer drivers, particularly when driving the A-dolly configuration, would be expected to experience cautionary-tored-line workload levels during which performance could decline rapidly to below acceptable levels.

Figure 2.

Configuration carryover effect for perceived workload.

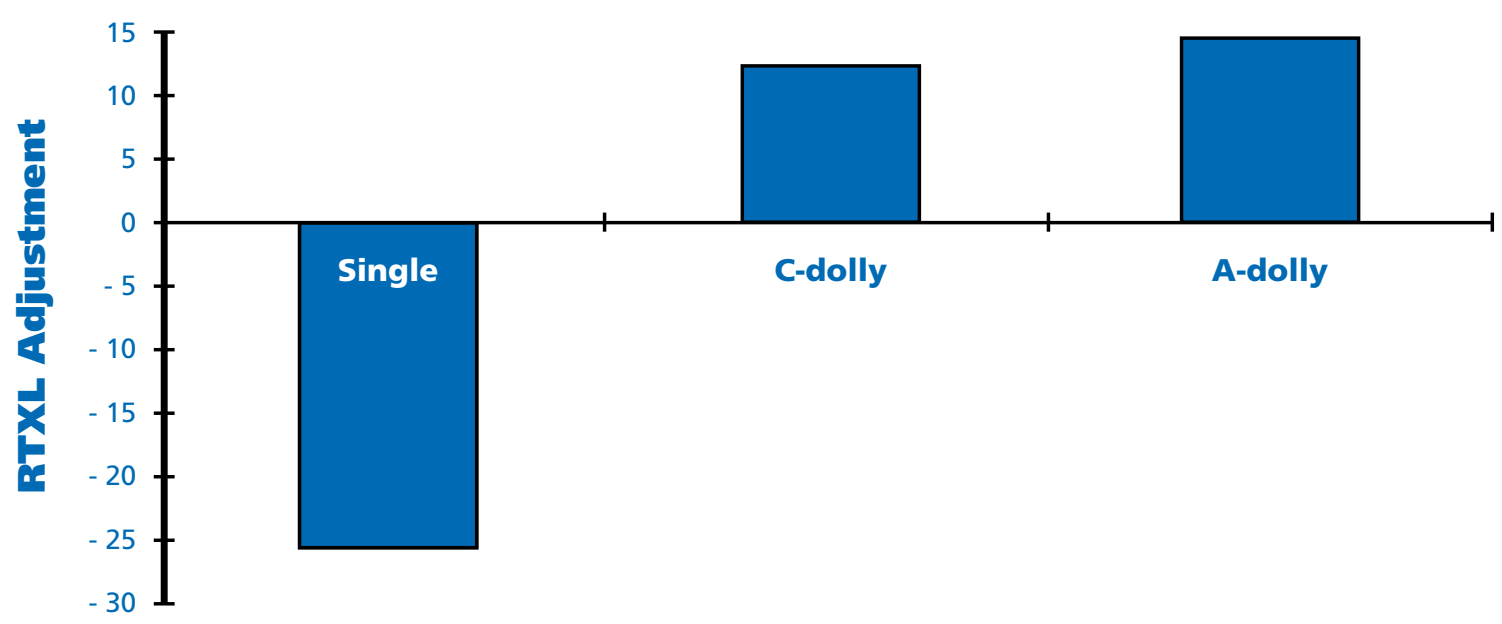


Researcher

Battelle Human Factors

Transportation Center, Seattle, Washington 98105. Contract

No. DTNH22-92-D-07001

\section{Distribution}

This Tech Brief is being distributed according to a standard

distribution. Direct distribution

is being made to the Service

Centers and Divisions.

\section{Avallability}

The study final report will be available from the National

Technical Information Service,

Telephone: (703) 605-6000

\section{Key Words}

truck driver, fatigue, alertness, CMV driver, vigilance, LongerCombination Vehicle (LCV), Multi-Trailer Combination Vehicle.

\section{Notice}

This Tech Brief is disseminated under the sponsorship of the

Department of Transportation

in the interest of information

exchange. The Tech Brief

provides a synopsis of the study's final publication. The

Tech Brief does not establish policies or regulations, nor does it imply USDOT endorsement of the conclusions or recommendations. The U.S. Government assumes no liability for its contents or their use.

\section{Web Site}

All FMCSA Tech and Analysis

Briefs may be accessed at:

http://www.fmcsa.dot.gov.

Please send us your comments

or suggestions about the

Tech/Analysis briefs.

\section{Technical Writer}

Hallie Magyar, Avalon

Integrated Services.

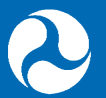

U.S. Department of Transportation Federal Motor Carrier Safety Administration

July 2000

Publication No. FMCSA-MCRT-00-012
Figure 3.

Conceptual overview of results.

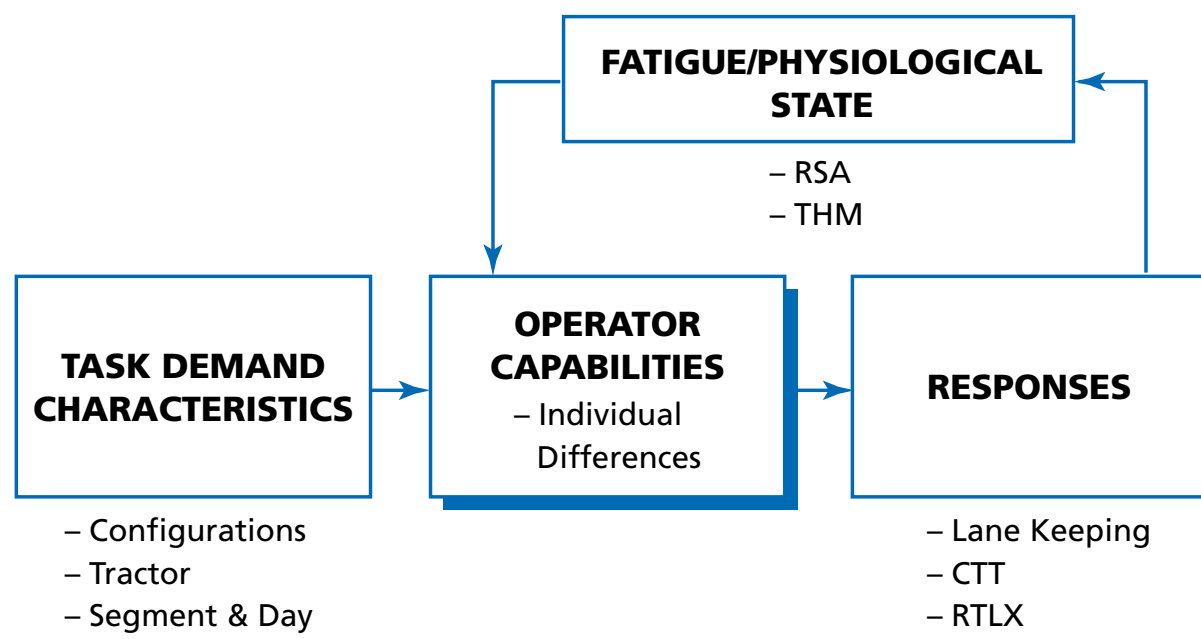

Together these considerations indicate that triple-trailer drivers generally would be expected to maintain a higher probability of safe operation with the Super C-dolly configuration than with the standard A-dolly

configuration (see Figure 3).

\section{Conclusions}

Three general conclusions regarding configuration effects can be drawn from the study results:

1. Configurations and other task demands consistently affected performance, subjective workload, and fatigue/physiological state following an SCA pattern. The A-dolly configuration resulted in the highest level of workload and fatigue, the C-dolly configuration resulted in the next highest level, and the single-trailer configuration resulted in the lowest level of workload and fatigue.

2. Driver differences were prominent in all analyses representing between 32 and 51 percent of the relative mean squares for lane-keeping and workload variables. The results indicate that some drivers are challenged by the driving task and support adoption of means to reduce this challenge, particularly when driving triples.

3. Configurations also have consistent carryover effects of physiological effects, driver subjective workload, and lane keeping performance, and imply that triple-trailer drivers generally would be expected to maintain a higher probability of safe operation with the Super C-dolly configuration than with the standard A-dolly configuration. 